\title{
Partitioned Computation for Fluid-structure Interaction with Rigid Body Motion
}

\author{
Asim Timalsina ${ }^{1}$, Gene $\mathrm{Hou}^{2}$ and Jin Wang ${ }^{3 *}$ \\ ${ }^{1}$ Department of Mathematics and Statistics, Old Dominion University, Norfolk, VA 23529 \\ ${ }^{2}$ Department of Mechanical and Aerospace Engineering, Old Dominion University, Norfolk, VA 23529 \\ ${ }^{3}$ Department of Mathematics, University of Tennessee at Chattanooga, Chattanooga, TN 37403 \\ Email: Jin-Wang02@utc.edu
}

\begin{abstract}
We present a new computational framework for fluid-structure interaction problems that involve rigid body motion. Based on a partitioned strategy, the fluid and structural dynamics are computed separately, and the solutions communicate at the interface. The immersed boundary method is employed to handle the fluid-structure interaction, and the direct-forcing technique is utilized to calculate the interaction force. In particular, the rigid body motion is directly and explicitly incorporated into the formulation and computation of the structural dynamics. We demonstrate our methodology by numerically simulating the motion of single and multiple rigid circular discs interacting with nonlinear viscous flow.
\end{abstract}

Keywords: Fluid-structure interaction; immersed boundary method; rigid body

\section{Introduction}

There have been a large number of numerical methods developed for the computation of fluid-structure interaction (FSI) problems $[4,10]$. Among these the immersed type methods, originated from the immersed boundary method [14] invented by Peskin in 1970s, have achieved much success. Typically, a method in this class represents the effects of the immersed solid structure by adding a forcing term on the right-hand side of the fluid momentum equation. As a result, the fluid motion can be computed on fixed Cartesian grids, which eliminates the need of costly re-meshing procedures. Naturally, the immersed type methods (and many other FSI algorithms) are suitable for the computation of elastic (or, deformable) solids interacting with fluid flows. For example, in the immersed boundary method, the FSI force is evaluated by using the Fréchet derivative of the elastic energy [14]. In the immersed finite element method, part of the force is calculated based on the stress tensor of the elastic structure that is determined by the material property [26].

In many FSI applications, however, it is necessary to consider rigid (or nearly rigid) body motion. On one hand, the motion of a rigid solid body is always characterized by translation (change of the linear position) and rotation (change of the angular position), with only three degrees of freedom in 2D space and six in 3D space. When interacting with fluid flow, it does not exhibit (possibly, complex) load deformation as does an elastic structure; thus a constitutive equation is not needed in the FSI computation of rigid body motion. On the other hand, the solid body may go through large translation and rotation under the rigidity constraint which may impose a challenge in the numerical simulation of FSI problems, especially for using the immersed type methods.

A common approach to treat the rigid body motion using an immersed type algorithm is to assign large elastic modulus, which can make the system very stiff and easily cause numerical instability [12]. Consequently, it becomes necessary to employ an implicit time marching method, or an explicit method with an extremely small time stepsize, either of which reduce the efficiency of the algorithm. A similar approach is based on the penalty method [5,9], where some stiff springs are attached to a solid structure so that even a small distortion of the solid will yield a spring force that tends to restore the solid to its original shape. Goldstein et al. [7] and Saiki and Biringen [17] employed a feedback force to represent a stationary rigid body, where the no-slip condition is satisfied by ensuring that the fluid velocity is zero at the interface under such a force. A drawback of this method is that the feedback force may induce spurious oscillations. The direct-forcing approach, proposed by Mohd-Yusof [13], imposes the no-slip 
constraint in the fluid momentum equation at the interface, so that the FSI force can be directly evaluated with the incorporation of the known structural interfacial velocity. The direct-forcing approach overcomes the numerical stiffness encountered by those afore-mentioned techniques and allows relatively large time step sizes to be employed $[8,23]$.

Generally speaking, to achieve high accuracy and maintain robust stability in FSI computation, a monolithic approach $[11,16]$ could be utilized where a unified system represents the entire FSI problem and a single algorithm solves the fluid and structural dynamics simultaneously. However, a monolithic approach typically demands large computational effort, and it may require substantial resources and expertise to develop and implement such an algorithm. Hence, a partitioned approach $[18,24]$ is more commonly employed that treats the fluid and the structure as two systems which can be computed separately in their respective domains and then connected at the interface. Compared to the monolithic counterpart, a partitioned algorithm offers improved efficiency, ease of implementation, and significant flexibility in taking advantage of well developed disciplinary solvers for the fluid and structural dynamics [10]. Recently, a partitioned approach based on the immersed framework and the direct-forcing technique was proposed for FSI computation that mainly involve elastic structures [21]. The method can deal with various shapes and material types of immersed structures with realistic constitutive laws, and allow the fluid and structural motion to be handled separately by their respective solvers.

In the present paper, we extend the method in [21] to deal with rigid body motion in FSI. We keep the main feature of the partitioned approach; i.e., the fluid dynamics and the structural dynamics are computed separately, and the solutions only communicate at the interface, and we utilize the direct-forcing technique for the calculation of the interaction force. Our special focus is on the structural part, where we explicitly describe the rigid body motion through the momentum equations of the solid structure. In particular, we demonstrate our methodology by considering the motion of rigid circular discs interacting with nonlinear viscous flow described by Navier-Stokes equations.

We organize this paper as follows. In Section 2 we describe the mathematical formulation, particularly for the rigid body treatment. In Section 3 we describe our numerical algorithm for the FSI computation. In Section 4 we present several numerical examples, including stationary and moving, single and multiple, rigid discs. In Section 5 we draw the conclusion and make brief discussion.

\section{Mathematical Equations}

We let $\Omega$ denote the entire physical domain of our interest, and let $\Omega^{f}$ denote the fluid domain and $\Omega^{s}$ denote the structure domain. Meanwhile, let $\rho$ denote the density, $\mathbf{V}$ the velocity, $\sigma$ the stress, and $\mathbf{G}$ the external force (such as gravity), in an appropriate domain. The equations of motion for the fluid and the structure are then given symbolically by

$$
\begin{aligned}
& \rho^{f} \frac{d \mathbf{V}^{\mathbf{f}}}{d t}=\nabla \cdot \sigma^{\mathbf{f}}+\mathbf{G}^{\mathbf{f}}, \quad \text { in } \Omega^{f} \\
& \rho^{s} \frac{d \mathbf{V}^{\mathbf{s}}}{d t}=\nabla \cdot \sigma^{\mathbf{s}}+\mathbf{G}^{\mathbf{s}}, \quad \text { in } \Omega^{s}
\end{aligned}
$$

Here and in what follows, the superscripts $f$ and $s$ refer to quantities associated with the fluid and structure, respectively. Furthermore, let $\Gamma$ denote the fluid-structure interface. The motion of the fluid and structure has to abide by the no-slip condition:

$$
\mathbf{V}^{\mathbf{f}}=\mathbf{V}^{\mathbf{s}}, \quad \text { on } \Gamma
$$

We consider a viscous and incompressible fluid. Then equation (1 ) for the motion of the fluid can be recast as the incompressible Navier-Stokes equations

$$
\begin{array}{r}
\rho^{f}\left(\frac{\partial \mathbf{V}^{\mathbf{f}}}{\partial t}+\mathbf{V}^{\mathbf{f}} \cdot \nabla \mathbf{V}^{\mathbf{f}}\right)+\nabla p-\mu \nabla^{2} \mathbf{V}^{\mathbf{f}}=\mathbf{G}^{\mathbf{f}}, \text { in } \Omega^{\mathrm{f}} \\
\nabla \cdot \mathbf{V}^{\mathbf{f}}=0, \text { in } \Omega^{\mathrm{f}}
\end{array}
$$

where $p$ denotes the pressure and $\mu$ denotes the dynamic viscosity of the fluid. 
The equation of motion for a general structure can be obtained by integrating equation (2) over the entire body. However, in the case of a rigid body, $\nabla \cdot \sigma^{\mathbf{s}}=0$ and the velocity can be described explicitly in terms of the independent degrees of freedom related to the rigid body translation and rotation. The detailed derivation of the equation of motion for a rigid body, particularly in the context of a rigid circular disc, is given in the following section.

\section{$2.1 \quad$ Rigid Body Motion}

We first describe the equation of motion for a free rigid body. Figure 1 shows a rigid body occupying the domain, $\Omega^{s}$. Two coordinate systems are used here to uncouple the translation and rotation that determine the motion of the rigid body. One is the global coordinate system, $X Y Z$, which is fixed on the ground. The other is the body-fixed coordinate system, $x^{\prime} y^{\prime} z^{\prime}$, whose origin is placed at point O. The movement of an arbitrary point, $\mathrm{P}$, in the rigid body can be measured by the relative motion between the body-fixed coordinate system and the global coordinate system. That is, the position vector of point $\mathrm{P}$ in the body, $\mathbf{r}$, is defined in terms of the global coordinate system as

$$
\mathbf{r}=\mathbf{R}+A \mathbf{r}^{\prime}
$$

where $\mathbf{R}$ is the position vector of the origin of the body-fixed coordinate system, $\mathbf{r}^{\prime}$ is the position vector of the point in the body with respect to the body-fixed co-ordinate system, which is in terms of the local coordinate system, and $A$ is the transformation matrix in terms of the angular displacements of the body. Thus, according to the presented model, the degrees of freedom of the rigid body are made of those describing the motion of the body-fixed coordinates; i.e., the translation, $\mathbf{R}$, and the orientation, $\theta$, of the body-fixed coordinate system.

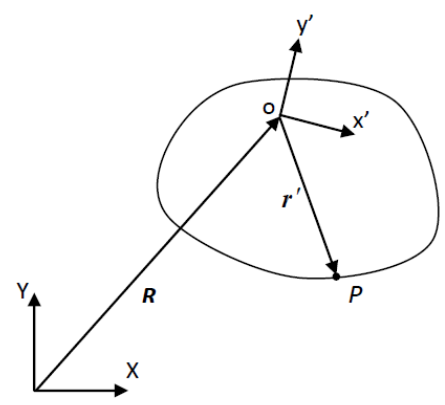

Figure 1: Coordinate systems for a rigid body.

The matrix equation of the motion of a rigid body can be symbolically expressed by

$$
M \ddot{\mathbf{q}}-\mathbf{f}^{s}(\mathbf{q}, \dot{\mathbf{q}}, t)=0
$$

where the unknown vectors and their time derivatives are given as

$$
\mathbf{q}^{T}=\left(\begin{array}{ll}
\mathbf{R}^{T} & \theta^{T}
\end{array}\right), \quad \dot{\mathbf{q}}^{T}=\left(\begin{array}{ll}
\dot{\mathbf{R}}^{T} & \mathbf{w}^{T}
\end{array}\right), \quad \ddot{\mathbf{q}}^{T}=\left(\begin{array}{ll}
\ddot{\mathbf{R}}^{T} & \boldsymbol{\alpha}^{T}
\end{array}\right)
$$

in which $\mathbf{w}$ is the angular velocity and $\boldsymbol{\alpha}$ is the angular acceleration. Here and in what follows, the single dot and double dots refer to the first and second time derivatives, respectively. The mass matrix, $M$, and the force vector, $\mathbf{f}^{s}$, can be further spanned as follows, based on their corresponding degrees of freedom:

$$
M=\left[\begin{array}{cc}
M_{R R} & M_{R \theta} \\
M_{\theta R} & M_{\theta \theta}
\end{array}\right] \quad \mathbf{f}^{s}=\left[\begin{array}{c}
\mathbf{f}_{R} \\
\mathbf{f}_{\theta}
\end{array}\right]
$$


Here $\mathbf{f}^{s}$ refers to the structural body forces that drive the motion of the structure.

For the case when the origin of the body-fixed coordinate system, point $\mathrm{O}$ in Figure 1, lies at the centroid of the body, one has $M_{r \theta}=M_{\theta R}=0$. Thus the mass matrix can be written as

$$
M=\left[\begin{array}{cc}
M_{R R} & 0 \\
0 & M_{\theta \theta}
\end{array}\right]=\left[\begin{array}{cc}
M & 0 \\
0 & I_{\tilde{r}^{\prime} \tilde{r}^{\prime}}
\end{array}\right]
$$

and the force vector as

$$
\mathbf{f}^{s}=\left[\begin{array}{c}
\mathbf{f}_{R} \\
\mathbf{f}_{\theta}
\end{array}\right]=\left[\begin{array}{c}
\mathbf{F}_{O}+\nu \mathbf{f}_{b} \\
-\widetilde{\mathbf{w}}^{\prime} I_{\tilde{r}^{\prime} \tilde{r}^{\prime}} \mathbf{w}^{\prime}+\mathbf{T}_{O}^{\prime}
\end{array}\right]
$$

Specifically, the equations of motion for a rigid body with its body-fixed coordinate system being placed at its centroid are given by

$$
\left\{\begin{array}{l}
M \ddot{\mathbf{R}}=\mathbf{F}_{O}+\nu \mathbf{f}_{b} \\
I_{\tilde{r}^{\prime} \tilde{r}^{\prime}} \boldsymbol{\alpha}^{\prime}=-\widetilde{\mathbf{w}^{\prime}} I_{\tilde{r}^{\prime} \tilde{r}^{\prime} \mathbf{w}^{\prime}+\mathbf{T}_{O}^{\prime}}
\end{array}\right.
$$

where we have introduced the tilde notation, say, $\widetilde{\boldsymbol{\beta}}$, to denote a skew matrix associated with a vector, say, $\boldsymbol{\beta}=\left(\begin{array}{lll}\beta_{x} & \beta_{y} & \beta_{z}\end{array}\right)^{T}$, by

$$
\widetilde{\boldsymbol{\beta}}=\left[\begin{array}{ccc}
0 & -\beta_{z} & \beta_{y} \\
\beta_{z} & 0 & -\beta_{x} \\
-\beta_{y} & \beta_{x} & 0
\end{array}\right]
$$

Note that in equation (8), $M$ is a $3 \times 3$ diagonal matrix with the value of the mass, $\nu$ is the volume of the body and $I_{\tilde{r}^{\prime}} \tilde{r}^{\prime}$ is the mass moment of inertia of the body which is defined as

$$
\begin{aligned}
I_{\tilde{r}^{\prime} \tilde{r}^{\prime}} & =\int_{\Omega} \rho \tilde{\mathbf{r}}^{\prime} \tilde{\mathbf{r}}^{\prime} \mathrm{d} v \\
& =\int_{\Omega} \rho\left[\begin{array}{ccc}
z^{\prime 2}+y^{\prime 2} & -x^{\prime} y^{\prime} & -x^{\prime} z^{\prime} \\
-y^{\prime} x^{\prime} & z^{\prime 2}+x^{\prime 2} & -y^{\prime} z^{\prime} \\
-z^{\prime} x^{\prime} & -z^{\prime} y^{\prime} & y^{\prime 2}+x^{\prime 2}
\end{array}\right] \mathrm{d} v
\end{aligned}
$$

For those terms on the right-hand side of equation (8), they are associated with the external forces; $\mathbf{F}_{O}$ is the resultant force applied at the origin of the body-fixed coordinate system, point $\mathrm{O}$, in terms of the global coordinate system, $\nu \mathbf{f}_{b}$ is the body force due to the weight of the body and $\mathbf{T}_{O}^{\prime}$ is the resultant torque produced by the external forces, applied at point $\mathrm{O}$ in terms of the body-fixed coordinate system. In addition, $-\widetilde{\mathbf{w}^{\prime}} I_{\tilde{r}^{\prime}} \tilde{r}^{\prime} \mathbf{w}^{\prime}$ is the centrifugal force induced by the angular velocity $\mathbf{w}^{\prime}$, in terms of the local coordinate system.

In this study, the rigid body is discretized into finite elements. The rigid body mass, $M$, as well as the moment of inertia, $I_{\tilde{r}^{\prime}} \tilde{r}^{\prime}$, defined in the equation above will be integrated over elements. The distributed pressure and friction induced by the fluid flow are integrated over the body boundary and converted into point loads imposed over the boundary nodes of the meshed domain. These equivalent nodal forces are then summed together to form the resultant force $\mathbf{F}_{O}$ and the torque $\mathbf{T}_{O}^{\prime}$.

\subsection{Equations of Motion for a Circular Disc}

Now we focus our attention on the motion of a circular, rigid disc in two dimensional space. The coordinate system of a circular disc is the same as described above with the origin of the body-fixed coordinate system being placed at its center. The disc itself is subject to the gravity along the negative $y$ direction and the circumference of the disc is subject to the distributed fluid pressure and the surface friction. Since our problem is two dimensional with three degrees of freedom and the circular disc is symmetric with respect to the body-fixed coordinate system, equation (8) can be simplified as

$$
\left[\begin{array}{ccc}
m & 0 & 0 \\
0 & m & 0 \\
0 & 0 & I_{\tilde{z}^{\prime} \tilde{z}^{\prime}}
\end{array}\right]\left[\begin{array}{c}
\ddot{\mathbf{R}}_{x} \\
\ddot{\mathbf{R}}_{y} \\
\ddot{\theta}
\end{array}\right]=\left[\begin{array}{c}
\mathbf{F}_{o x}+\nu \mathbf{f}_{b x} \\
\mathbf{F}_{o y}+\nu \mathbf{f}_{b y} \\
\mathbf{T}_{o z}
\end{array}\right] \equiv\left[\begin{array}{c}
f_{x} \\
f_{y} \\
T_{z}
\end{array}\right]
$$


The mass, the volume and the mass moment of inertia, can all be obtained as the sum of integrals, each of which is numerically evaluated in the associated quadrilateral element $\Omega_{i}$. For instance, the mass moment of inertia is calculated as

$$
I_{\tilde{z}^{\prime} \tilde{z}^{\prime}}=\sum \int_{\Omega} \rho\left(y^{\prime 2}+x^{\prime 2}\right) \mathrm{d} v
$$

The distributed loads due to surface pressure and friction can also be lumped into equivalent nodal forces based upon the definition of a quadrilateral finite element. With these computed boundary nodal forces, the induced, resultant forces $\left(\mathbf{F}_{o x} \mathbf{F}_{o y}\right)$ and the torque $\mathbf{T}_{o z}$ can then be conveniently calculated.

\section{Numerical Algorithm}

The fluid equations (i.e., the incompressible Navier-Stokes equations) are solved in time by using a projection method [14], which is essentially a predictor-corrector procedure. At the predictor step, an intermediate solution is computed for the velocity and pressure, and then the intermediate velocity is projected into the divergence-free subspace to obtain the corrected solution. The structural equations are discretized using finite elements, and then solved in time by a one-step implicit Newmark method [1]. Since the structure is a rigid body, calculation of the strain and stress is not needed.

The fluid-structure interaction is handled by using the immersed boundary method [14], which adds a forcing term $\mathbf{f}_{\mathbf{1}}$ to the right-hand side of the fluid momentum equation:

$$
\rho^{f}\left(\frac{\partial \mathbf{V}^{\mathbf{f}}}{\partial t}+\mathbf{V}^{\mathbf{f}} \cdot \nabla \mathbf{V}^{\mathbf{f}}\right)+\nabla p-\mu \nabla^{2} \mathbf{V}^{\mathbf{f}}=\mathbf{G}^{\mathbf{f}}+\mathbf{f}_{\mathbf{1}}
$$

Here $\mathbf{f}_{\mathbf{1}}$ represents the fluid-structure interaction force acting on the fluid motion. We use the direct forcing approach, originally proposed by Mohd-Yusof [13], to calculate this force. Specifically, we evaluate the force directly from equation (10) at the Lagrangian grid points, using the known structural velocity $\mathbf{V}^{\mathbf{s}}$, based on the procedure described in [23]. At the time step $n+1$, our forcing term $\mathbf{F}_{\mathbf{1}}$ on the Lagrangian grids takes the form

$$
\mathbf{F}_{\mathbf{1}}{ }^{n+1}= \begin{cases}\rho^{f}\left[\frac{\mathbf{V}^{\mathbf{s}}-\mathbf{V}^{n}}{\Delta t}+((\mathbf{V} \cdot \nabla) \mathbf{V})^{n}\right]+\nabla p^{n}-\mu \nabla^{2} \mathbf{V}^{n}-\mathbf{G}^{\mathbf{f}} & \text { on } \Gamma^{s} \\ \mathbf{0} & \text { elsewhere }\end{cases}
$$

In the above formulation, the fluid variables are evaluated on the Lagrangian grids on $\Gamma^{s}$. Once computed, the force $\mathbf{F}_{\mathbf{1}}$ is passed to the structure code acting as the force load, and the structural displacement and velocity fields are updated accordingly. Meanwhile, the force $\mathbf{F}_{\mathbf{1}}$ is interpolated, through a discrete delta function, to the Eulerian grid so as to solve the Navier-Stokes equations and update the fluid motion. Once the interpolation is completed, the forcing term $\mathbf{f}_{\mathbf{1}}$ on the right-hand side of equation (10) is obtained.

Our FSI algorithm, which integrates the immersed boundary method and the direct-forcing technique into a partitioned framework, can be summarized below. Assume that at $t=t_{n}$, the structural velocity $\mathbf{V}^{s, n}$, the fluid velocity $\mathbf{V}^{f, n}$ and pressure $p^{n}$, and the interface position $\Gamma^{n}$ are known. The following procedure computes the solution at $t=t_{n+1}$.

1. Compute $\mathbf{V}^{f, n},((\mathbf{V} \cdot \nabla) \mathbf{V})^{f, n}, \nabla^{2} \mathbf{V}^{f, n}$, and $\nabla p^{n}$ on the interface through interpolation from the Eulerian grids to the Lagrangian grids.

2. Calculate the force $\mathbf{F}_{\mathbf{1}}{ }^{n+1}$ on the Lagrangian grids using the direct-forcing approach (see equation 11).

3. Distribute the force from the interface to the fluid domain to obtain $\mathbf{f}_{\mathbf{1}}{ }^{n+1}$, and compute the NavierStokes equation by the projection method.

4. Pass the force $-\mathbf{F}_{\mathbf{1}}{ }^{n+1}$ as the load to the structural solver, and compute the structural motion at $t=t_{n+1}$; particularly, obtain the boundary displacement $\mathbf{W}^{n+1}$ and velocity $\mathbf{V}^{s, n+1}$.

5. Update the fluid-structure interface with the updated structural displacement obtained from the structure code; e.g.,

$$
\Gamma^{n+1}=\Gamma^{n}+\mathbf{W}^{n+1}
$$

6. Pass the updated structural velocity $\mathbf{V}^{s, n+1}$ and interface location $\Gamma^{n+1}$ to the fluid solver and start the next step of computation. 


\section{Simulation Results}

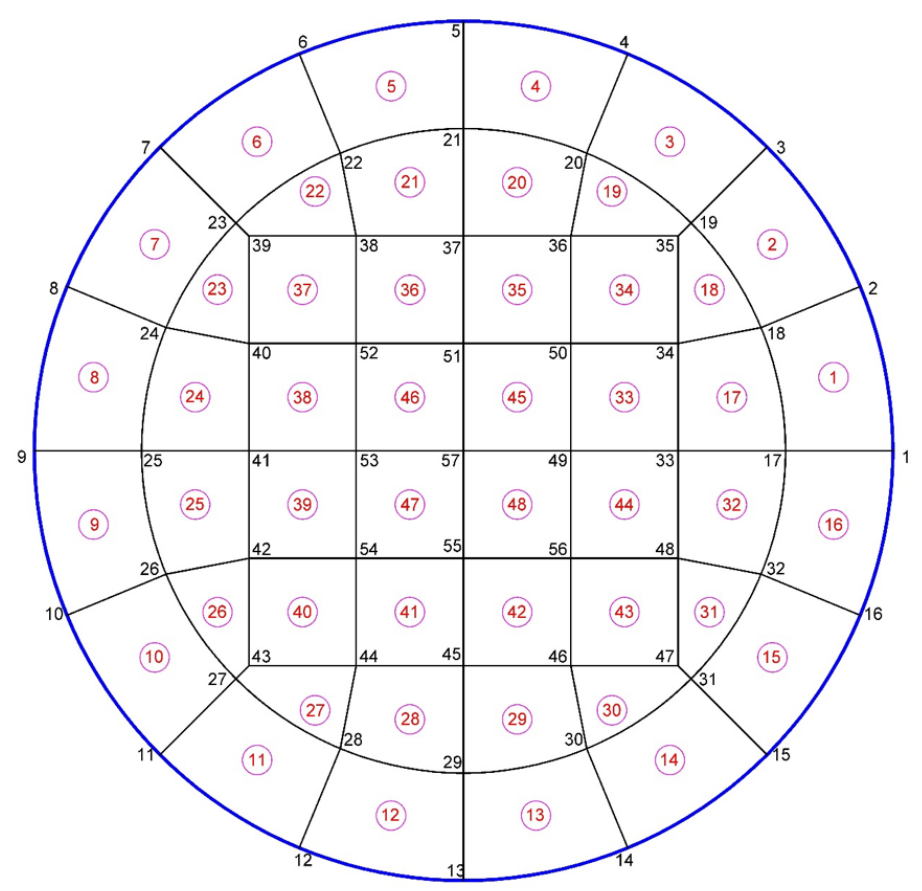

Figure 2: Finite element mesh on a rigid disc.

The computational domain is set to $[0,50] \times[0,50]$. The fluid flow is initialized as a uniform parallel flow from the left to the right, $\mathbf{u}_{0}=10 \mathrm{in} / \mathrm{s}$, with corresponding inflow and outflow boundary conditions. The density of the fluid is set as $\rho^{f}=0.5 \mathrm{lb} / \mathrm{in}^{3}$, and the dynamic viscosity $\mu=100 \mathrm{lb} /(\mathrm{in} \cdot \mathrm{s})$. A disc of radius 2 is placed at the center of the fluid domain, and its density is given as $\rho^{s}=10 \mathrm{lb} / \mathrm{in}^{3}$. Figure 2 shows the mesh of the disc that is discretized into 48 CQUAD elements with 57 grid points. The information between the fluid and the structure are exchanged at the 16 boundary nodes (Node 1 through 16 in the Figure 2). The position of the center $(\mathrm{x}, \mathrm{y}, \theta)$, velocity of the disc, and the boundary load are fed into the structure solver. In return, the fluid solver receives the velocity and the displacement of the disc.

\subsection{A Single Disc}

To begin, we first apply our algorithm to the canonical problem of viscous flow past a stationary cylinder (that is, a disc in 2D). This is a special case of our FSI problem where the motion of the disc is zero and the interface $\Gamma$ remains fixed. Figure 3 shows the streamline plots for two different values of the Reynolds number, denoted by Re. We observe that when $\mathrm{Re}=5$, the flow is parallel and symmetric, and no wakes form. In contrast, when $\mathrm{Re}=40$, a pair of vortices in wakes are observed in the tail region. We have also computed the drag coefficient and compared the result to those in the literature. The drag coefficient is calculated by using the formula:

$$
C_{D}=\frac{2 F_{x}}{\rho u^{2} D}
$$

where $F_{x}$ is the $x$ component of the interaction force acting on the disc, and D is the diameter of the disc. The comparison is presented in Table 1, and we see good agreement with previously published results.

Next, we run the simulation for a moving disc governed by the translational and rotational laws. A single disc of radius 2 is initially placed at the center of the domain, i.e. $(25,25)$. Under the effects of 


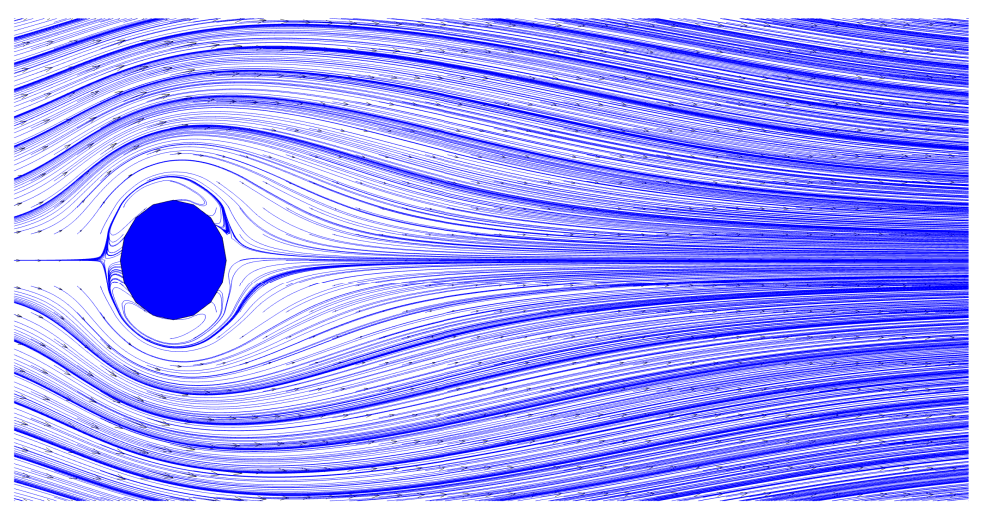

(a) $\mathrm{Re}=5$

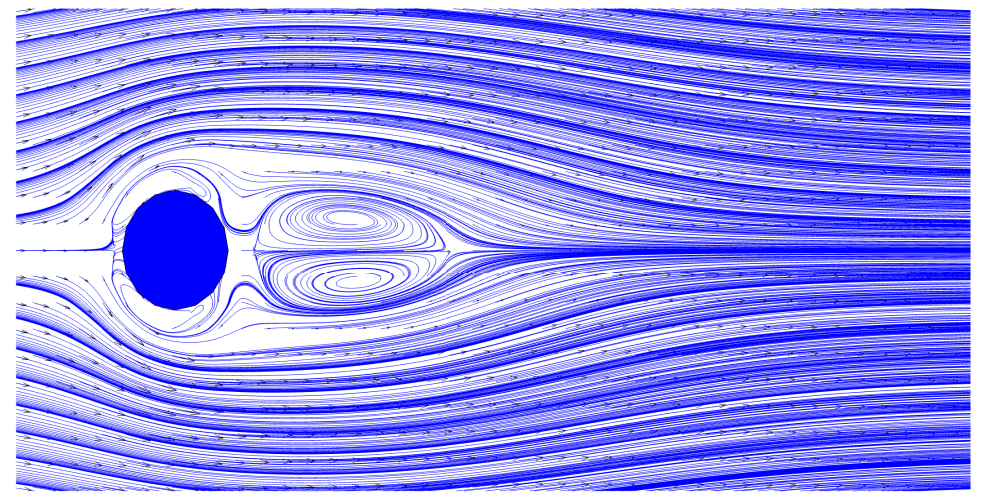

(b) $\operatorname{Re}=40$

Figure 3: Streamline visualization for viscous flow past a stationary disc. 
Table 1: Comparison of the drag coefficients.

\begin{tabular}{lc} 
Re=40 & $C_{D}$ \\
\hline Calhoun [2] & 1.62 \\
Dennis and Chang [3] & 1.52 \\
Fornberg [6] & 1.50 \\
Taira and Colonius [19] & 1.55 \\
Present & 1.50
\end{tabular}

the gravity and the background fluid flow, the disc moves in both the horizontal and vertical directions. Figure 4 shows the displacement of the center of the disc, relative to its initial position, as time goes from 0 to 1 . When there is no gravity, the disc would move in the $x$-direction only. As the gravity increases, the displacement in the negative $y$-direction increases. In Figure 5, the average torque force of the disc at $t=1$ is shown, in order to examine the rotation of the disc. We observe that when the the gravity is 0 , the torque becomes zero and there is no rotation for the disc. When the gravity is greater than 0 , the torque is positive, indicating that the disc rotates in the counter-clockwise direction. The reason is as follows. The falling disc moves from its initial center position to the lower edge of the channel. Thus, the flow underneath the disc moves faster than that above the disc, so that the pressure is higher on the lower edge of the disc than that on the upper edge. As a result, the falling of the disc slows down and the disc rotates in the counter-clockwise direction. As seen in the plot, the torque, and hence the rotation, of the disc gradually increase (in an approximately linear manner) as the gravity increases, since the distance between the disc and the bottom edge of the channel will decrease faster for a higher value of gravity. Figure 6 displays a snapshot of the disc at several times with gravity $g=1$. The disc moves to the right due to the impact of the fluid flow, and meanwhile moves downward due to the gravity.

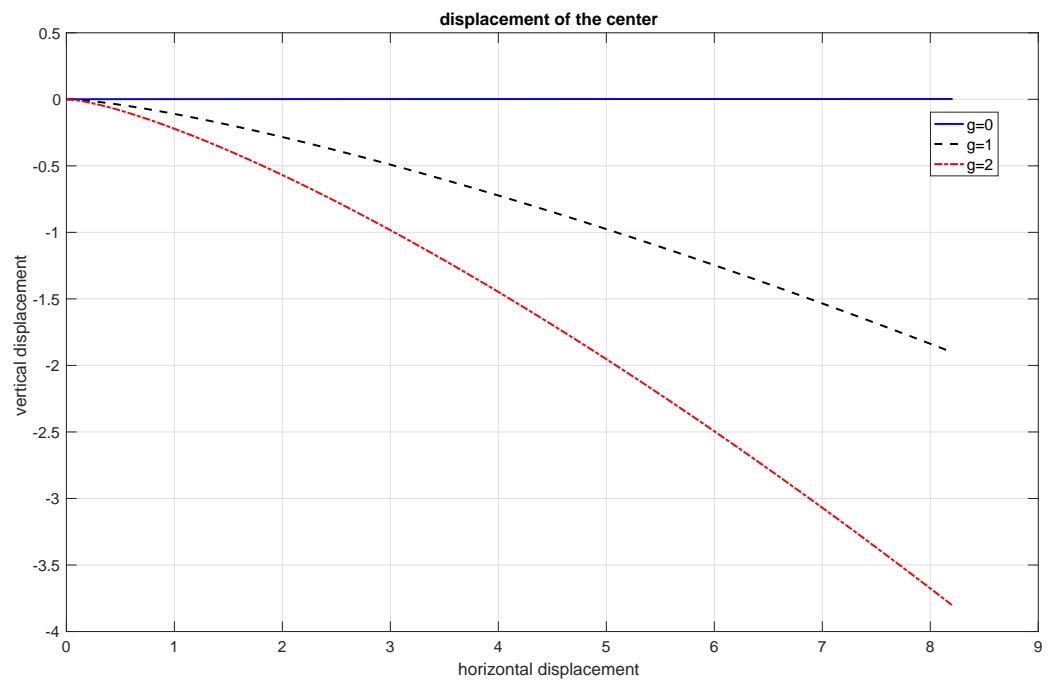

Figure 4: Displacement of the center of the disc for different gravity.

\subsection{Multiple Discs}

Now we extend our numerical simulation to the motion of multiple rigid discs interacting with fluid flows. Instead of trying to handle the general motion that can become highly complicated, we focus our 


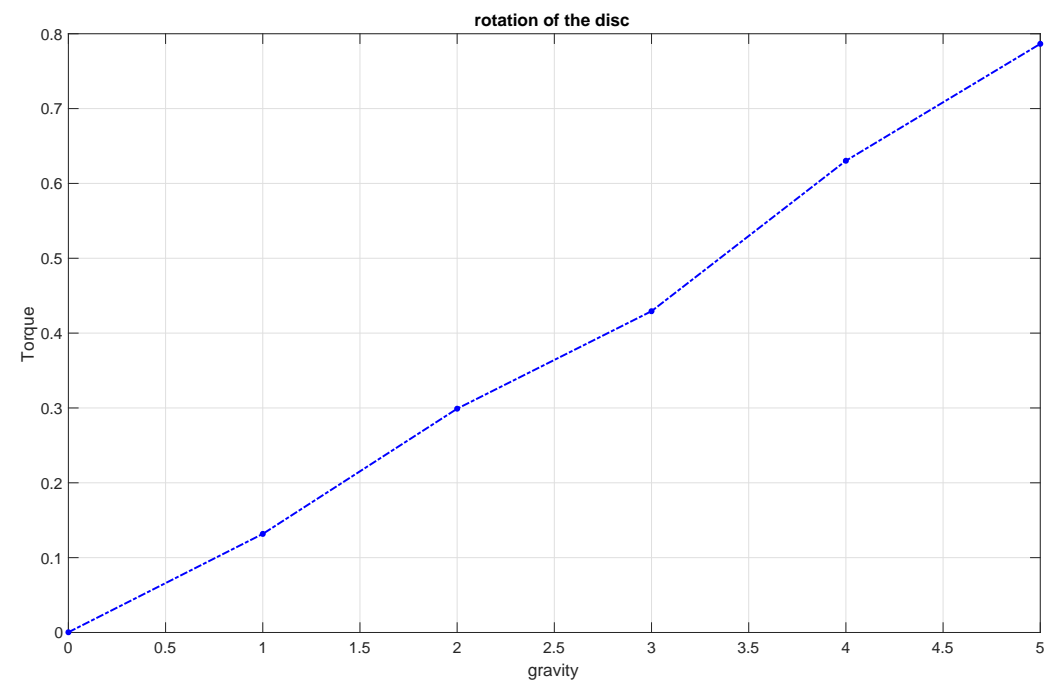

Figure 5: The average torque of the disc under varied gravity.

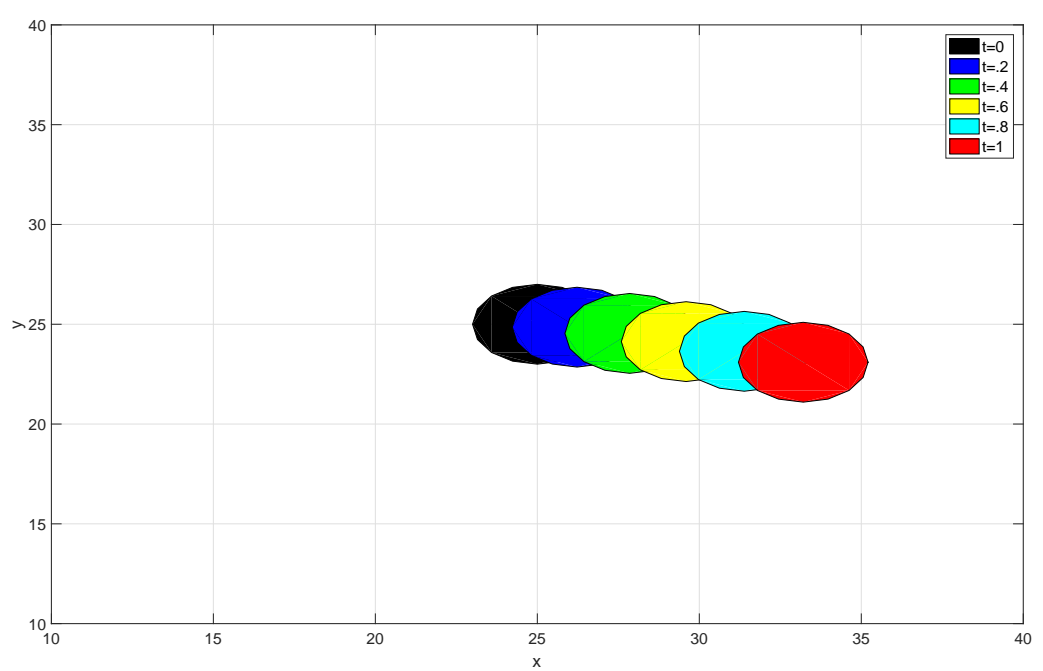

Figure 6: Snapshot of a moving disc at various times. 
attention on a special case concerned with the aggregation of the multiple solids. That is, we consider solid structures with strong adhesivity. Examples include bonding between surface-treated glass, metals, silicon or other semiconductor materials, adhesion between rigid particles and incompressible subtrate [25], adhesion between glass particles and polypropylene [20], as well as aggregation of cells and bonding of molecules or atoms on the microscale. In this study, these structures are treated in a simplistic manner as circular discs, where our focus is the movement and aggregation of these discs under the impact of the fluid flow. Specifically, we assume that whenever two such discs touch each other, they will adhere to each other and move as a union afterwards. To that end, we introduce an artificial adhesion force acting at the centers of each pair of discs, as described in [15]. Let the coordinates of the two centers be $\mathbf{X}_{k}(t)$ and $\mathbf{X}_{l}(t)$. The force is represented by Hooke's law with a constant spring stiffness $K_{a d h}$ :

$$
\mathbf{F}_{a d h}(l, t)= \begin{cases}K_{a d h} \frac{\left\|\mathbf{X}_{k}(t)-\mathbf{X}_{l}(t)\right\|-2 r}{\left\|\mathbf{X}_{k}(t)-\mathbf{X}_{l}(t)\right\|}\left(\mathbf{X}_{k}(t)-\mathbf{X}_{l}(t)\right) & \text { if }\left\|\mathbf{X}_{k}(t)-\mathbf{X}_{l}(t)\right\| \leq 2 r \\ \mathbf{0} & \text { otherwise }\end{cases}
$$

Here $r$ is the radius of each disc, and the adhesion force $\mathbf{F}_{a d h}$ is evaluated at each time step based on the positions of the discs. Once two discs touch each other, this force kicks in and attempts to keep the two discs as a union by maintaining the distance between the two centers at $2 r$. The adhesion force $\mathbf{F}_{a d h}$ is then incorporated into the final FSI force $\mathbf{F}_{\mathbf{1}}$.

The numerical procedure for the FSI computation described in Section 3 is modified to highlight the presence of multiple solid structures in our problem. Assume that there are $n$ discs and the initial distance between any two disc centers is at least $\epsilon$, where $\epsilon>2 r$.

- Calculate the total FSI forces, including the adhesion force, at the fluid-structure interface.

- Distribute the forces from the interface to the Eulerian fluid grids and solve the Navier-Stokes equations.

- Interpolate the fluid velocity from the background Eulerian grids to the interfacial Lagrangian grids for all discs.

- Calculate the center distance between each disc pair, and check if any two discs touch each other. If so, these two discs are treated as a union.

- With the boundary force, solve the structural equations for all discs. Note that the boundary force is treated as a constant while solving equation (8) numerically from timestep $t_{n}$ to $t_{n+1}$.

- Update the velocities and positions of all discs and proceed to the next time step.

In summary, whenever two discs collide, they attach on to one another and act as a union. The number of discs in a union may grow with time, as more discs touch each other, and a cluster (or, group) forms from this process.

We start with the simulation of three discs to illustrate the aggregation and movement process. We place the discs around the middle of the computational domain centered at $(20,25),(24.4,25)$, and $(23,29)$, and refer them to Disc 1 (shown in black), Disc 2 (shown in red), and Disc 3 (shown in blue), respectively. Once the three discs join each other and form a cluster, they move like a single structure. All the parameters used are the same as before along with the initial disc velocities of $(1,2),(-2,-2)$ and $(1,-1)$. Figure 7 shows the position of the discs at various times. Initially none of the discs are in contact with each other. A little before $t=0.2$, Disc 1 and Disc 2 touch one another. Very soon after that first contact, Disc 2 and Disc 3 also touch each other. Over the remaining course of the simulation, the cluster of the three discs continues to move along the flow as a single unit.

Finally, using the same rationale and methodology, we present a simulation case with 100 discs. They are initially placed randomly around the center region of the domain $[0,800] \times[0,800]$, with random initial velocities. Figures 8 displays the movement and aggregation of these discs over time. As seen in the plots, the discs form several clusters dictated by the position and velocity of each disc. Each cluster so formed moves as a single unit along the fluid flow. They are free to attach to another group of discs and form a bigger cluster, but may never detach from their original cluster. At $t=6$, we observe that these discs already form several large and small clusters and exhibit a heterogeneous pattern in their displacement. 

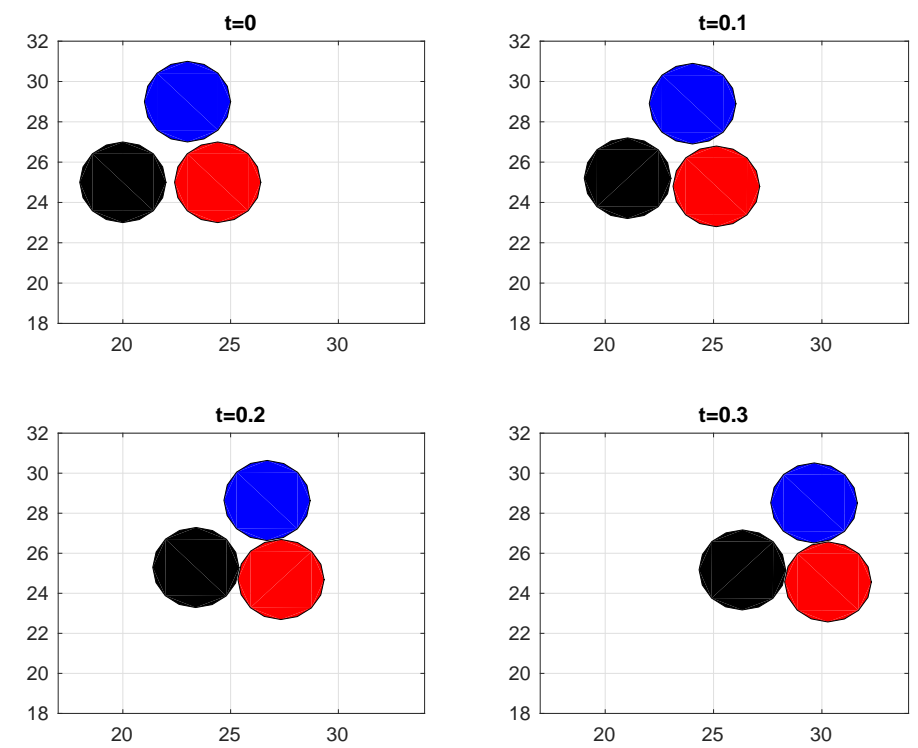

Figure 7: Motion of 3 discs at various times.
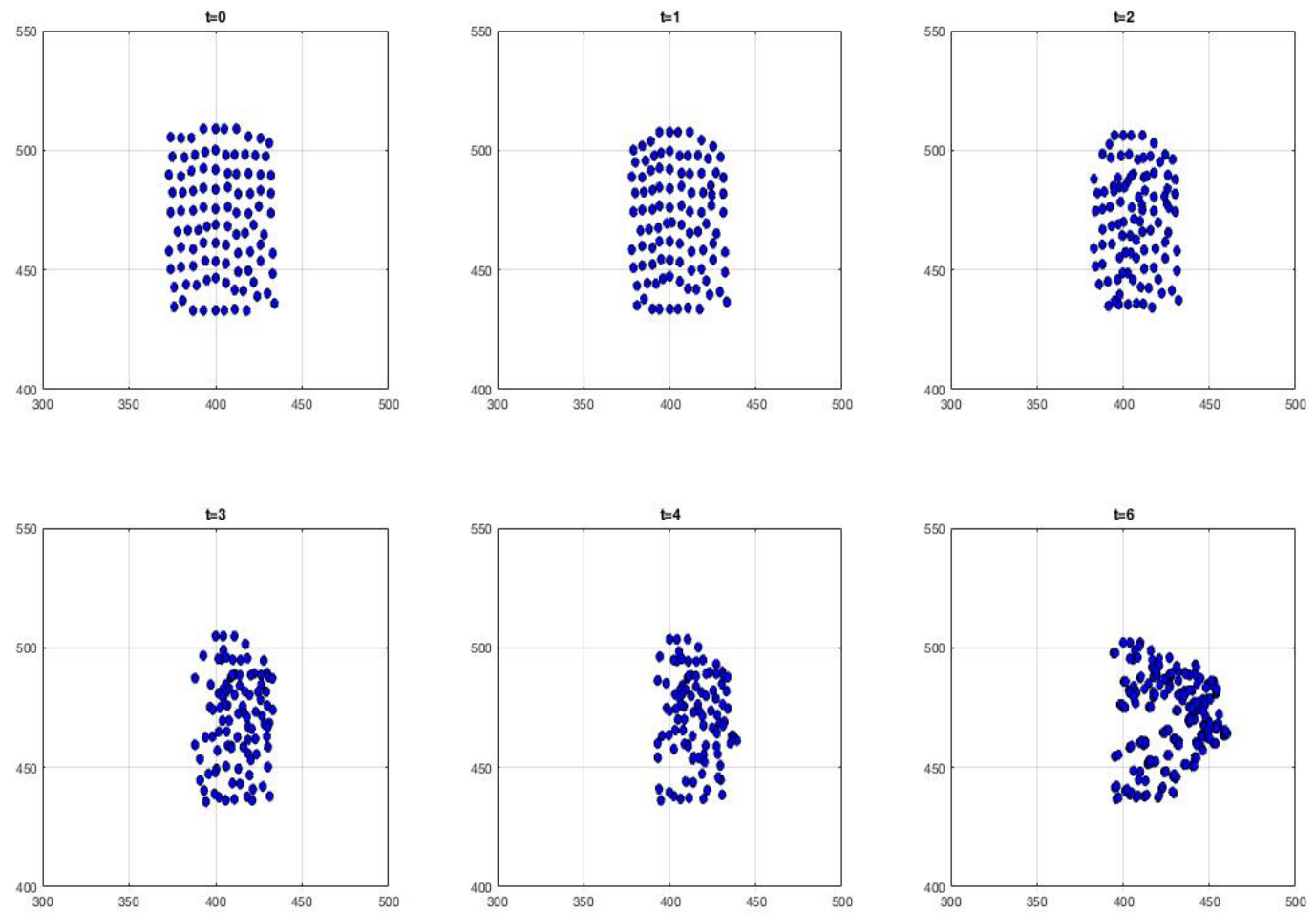

Figure 8: Motion of 100 discs at different times. These discs are randomly placed around the center of the domain at the initial time. At $t=6$, the discs already form several clusters and exhibit strong heterogeneity. 


\section{Conclusion}

In this paper we are concerned with the computation of fluid-structure interaction that involves rigid disc movement in Navier-Stokes flow. We have presented a new method that integrates the immersed type formulation and the direct-forcing technique, based on a partitioned framework. Unlike previous approaches that impose the rigidity constraint in a somewhat indirect way (e.g., through penalty or feed back forcing), our method directly incorporates the rigid body formulation in representing the structural dynamics. We have demonstrated the method through numerical simulation to FSI problems that involve both stationary and moving, both single and multiple, rigid discs interacting with fully nonlinear viscous fluid flow described by Navier-Stokes equations. Our numerical algorithm allows us to conduct simulation to these nontrivial FSI problems with as many as 100 discs. We have found that this method can accurately represent the rigid body motion in the FSI computation without introducing numerical instability.

We acknowledge that our current treatment of the structural interactions in the presence of multiple discs is simplistic and special. Our future work will explore more general strategy to handle the collision, repelling, and adhesion among different discs so that we may be able to simulate more realistic FSI problems of this type. We also plan to improve the efficiency of our algorithm and utilize high-performance computation so that we may conduct large simulations that could involve significantly more solid structures moving in the fluid flow and that could extend to three dimensional applications.

\section{References}

1. Bathe KJ, Nitikitpaiboon C and Wang X (1995), "A mixed displacement-based finite element formulation for acoustic fluid-structure interaction," Comput Struct 56: 225-237.

2. Calhoun D (2002), "A Cartesian grid method for solving the two-dimensional streamfunction-vorticity equations in irregular regions," J Comput Phys. 176: 231-275.

3. Dennis, SCR and Chang GZ (1970), "Numerical solutions for steady flow past a circular cylinder at Reynolds numbers up to 100," J Fluid Mech. 42: 471-489.

4. Dowell EH and Hall KC (2001), "Modeling of fluid-structure interaction," Ann Rev Fluid Mech. 33: 445-490.

5. Fogelson AL and Peskin CS (1988), "A fast numerical method for solving three-dimensional stokes equation in the presence of suspended particles," J Comput Phys. 79: 50-69.

6. Fornberg B (1980), "A numerical study of steady viscous flow past a circular cylinder," J Fluid Mech. 98: 819-855.

7. Goldstein D, Hadler R and Sirovich L (1993), "Modeling a no-slip flow boundary with an external force field," $J$ Comput Phys. 105: 354-366.

8. Guy RD and Hartenstine DA (2010), "On the accuracy of direct forcing immersed boundary methods with projection methods," J Comput Phys, 229: 2479-2496.

9. Hofler K and Schwarzer S (2000), "Navier-Stokes simulation with constraint forces: Finite-difference method for particle-laden flows and complex geometries," Phys Rev E 61: 7146-7160.

10. Hou G, Wang J and Layton A (2012), "Numerical methods for fluid-structure interaction - A review," Commun Comput Phys. 12: 337-377.

11. Hubner B, Walhorn E and Dinkler D (2004), "A monolithic approach to fluid-structure interaction using space-time finite elements," Comput Meth Appl Mech Eng. 193: 2087-2104.

12. Mittal R and Iaccarino G (2005), "Immersed boundary methods," Ann Rev Fluid Mech. 37: 239-261.

13. Mohd-Yusof J (1997), "Combined immersed boundary/B-splines method for simulations of flows in complex geometries," Center for Turbulence Research Annual Research Briefs, NASA Ames/Stanford University, pp. 317-327.

14. Peskin CS (2002), "The immersed boundary method," Acta Numer. 11: 479-517.

15. Rejniak KA and Dillon RH (2007), "A single cell-based model of the ductal tumour microarchitecture," Comput Math Methods Med. 8(1): 51-69.

16. Ryzhakov PB, Rossi R, Idelsohn SR and Onate E (2010), "A monolithic Lagrangian approach for fluid-structure interaction problems," Comput Mech. 46: 883-899.

17. Saiki EM and Biringen S (1996), "Numerical simulation of a cylinder in uniform flow: Application of a virtual boundary method," J Comput Phys. 123: 450-465.

18. Steindorf J and Matthies HJ (2000),"Numerical efficiency of different partitioned methods for fluid-structure interaction," ZAMM J Appl Math Mech. 80: 557-558.

19. Taira K and Colonius T (2007), "The immersed boundary method: a projection approach," J Comput Phys. 225: 2118-2137. 
20. Thio YS, Argon AS and Cohen RE (2004), "Role of interfacial adhesion strength on toughening polypropylene with rigid particles," Polymer, 45: 3139-3147.

21. Timalsina A, Hou G and Wang J (2017), "Computing Fluid-Structure Interaction by the Partitioned Approach with Direct Forcing," Commun Comput Phys. 21: 182-210.

22. Trepat X, Wasserman MR, Angelini TE, Millet E, Weitz DA, Butler JP, and Fredberg JJ (2009), "Physical forces during collective cell migration," Nature Phys. 5(6):426-430.

23. Uhlmann M (2005), "An immersed boundary method with direct forcing for the simulation of particulate flows," J Comput Phys. 209: 448-476.

24. Vierendeels J, Dumont K and Verdonck PR (2008), "A partitioned strongly coupled fluid-structure interaction method to model heart valve dynamics," J Comput Appl Math. 215: 602-609.

25. Yan S and He L (2012), "Adhesive force between a spherical rigid particle and an incompressible elastic substrate," Mech Mater 49: 66-71.

26. Zhang LT and Gay M (2007), "Immersed finite element method for fluid-structure interactions," J Fluids Struct. 23(6): 839-857. 